\title{
Retinal and Choroidal Optical Coherence Tomography Findings of Carotid Cavernous Fistula
}

\author{
ONUR INAM, YONCA OZKAN ARAT, GULIZ FATMA YAVAS, AND ANIL ARAT
}

- PURPOSE: To define the retinal and choroidal imaging findings of carotid cavernous fistula (CCF) including central foveal thickness, subfoveal choroidal thickness, choroidal vascularity index (CVI) parameters, and tortuosity indexes (TIs) as compared to a control group (CG). - DESIGN: Cross-sectional study.

- MATERIALS AND METHODS: The spectral domain enhanced-depth imaging optical coherence tomography images of 19 eyes of 19 consecutive patients with angiographically proven CCF and 19 eyes of 19 age- and sexmatched healthy control subjects were included. The patient group was divided according to CCF venous drainage pattern as anterior (A-CCF: draining into ophthalmic veins) and posterior (P-CCF: not draining into ophthalmic veins). The clinically affected eyes of the patient group, ipsilateral to the fistula, were included in the analysis.

- RESUlTS: There were 15 A-CCFs (78.9\%) and 4 P-CCFs $(21.1 \%)$. The mean SFCT of the A-CCF group $(395.21 \pm 111.69 \mu \mathrm{m})$ was significantly higher than those of the P-CCF $(246.84 \pm 94.12 \mu \mathrm{m})$ and CG groups $(280.79 \pm 111.36 \mu \mathrm{m})(P=.039$ and $P=.006$, respectively). The mean CVI of the A-CCF group was significantly higher than that of the CG $(68.97 \pm 4.81$ and $65.66 \pm 3.37$, respectively, $P=.033)$. The A-CCF group had significantly higher inferior, superior, and total venous $T I$ than the $C G$ group $(P=.001, P=.001$, and $P<.001$, respectively).

- CONCLUSION: In this first study investigating the CVI and TI in CCF patients, we demonstrated that SFCT, CVI, and TI could potentially be used to aid in the diagnosis of A-CCF. (Am J Ophthalmol 2019;206: 264-273. (C) 2019 Elsevier Inc. All rights reserved.)

\footnotetext{
AJO.com

Accepted for publication Jun 8, 2019.

From the Departments of Ophthalmology (O.I., Y.O.A., G.F.Y.) and Biophysics (O.I.), and the Division of Interventional Neuroradiology, Department of Radiology (A.A.), Hacettepe University, Ankara, Turkey.

Onur Inam is currently at the University of Health Sciences, Ulucanlar Eye Training and Research Hospital, Department of Ophthalmology, Ankara, Turkey.

Inquiries to Yonca Ozkan Arat, Department of Ophthalmology, Hacettepe University Hospital, Ankara, Turkey; e-mail: yoncaozkan@ hotmail.com
}

$\mathrm{T}$ HE CAROTID CAVERNOUS FISTULA (CCF) IS AN abnormal connection between the cavernous sinus and carotid arterial system. ${ }^{1}$ Clinical symptoms and presentation depend mainly on the location and pattern of venous drainage., ${ }^{2,3}$ In the anterior CCF (A-CCF), the fistula mainly drains into the ophthalmic vein, which may cause proptosis, chemosis, and vision loss. ${ }^{4}$ In the posterior CCF (P-CCF), drainage is mainly to the inferior petrosal sinus and then to the internal jugular vein. ${ }^{4}$ A-CCFs mostly present with congestion symptoms, whereas P-CCFs present mainly with pain, diplopia, and cranial nerve palsies. ${ }^{1}$ The most common associated ocular findings of A-CCF are exophthalmos, eyelid swelling, chemosis, dilated vessels, conjunctival injection, ophthalmoplegia, cranial nerve palsies, increased intraocular pressure, and venous congestion in iris and choroidal tissues. ${ }^{5-7}$

The choroid has the highest blood flow of any tissue in the body. ${ }^{8}$ The choroid is primarily composed of blood vessels surrounded by stromal tissue. ${ }^{9}$ Effects of CCF on choroidal tissue can be assessed with the emerging technologies that measure the thickness of the choroid, such as enhanced depth imaging (EDI) in spectral domain optical coherence tomography (SD-OCT). Increased subfoveal choroidal thickness was reported previously in the literature in only 3 cases of CCF. ${ }^{10-12}$ None of these published case reports answered the questions regarding the mechanism of thickening of the choroid in CCF patients. Thus, in addition to the definition of the morphological changes of choroid, we aimed to perform choroidal vascular analysis by quantifying the luminal ratio of the choroid using the technique previously described by Agrawal, namely, choroidal vascularity index (CVI), and by measuring the tortuosity index (TI) values of the infrared (IR) images of patients with CCF. ${ }^{13,14}$ To our knowledge, this is the first study in the literature to investigate both the morphology and the vascularity of the choroid in patients with CCF using thickness parameters as well as choroidal vascularity and tortuosity indices measured by the spectral domain enhanced-depth imaging optical coherence tomography (SD-EDI-OCT). These parameters of SD-EDI-OCT may have a role in the diagnosis of CCF, differentiating it from conditions with similar presentation. 


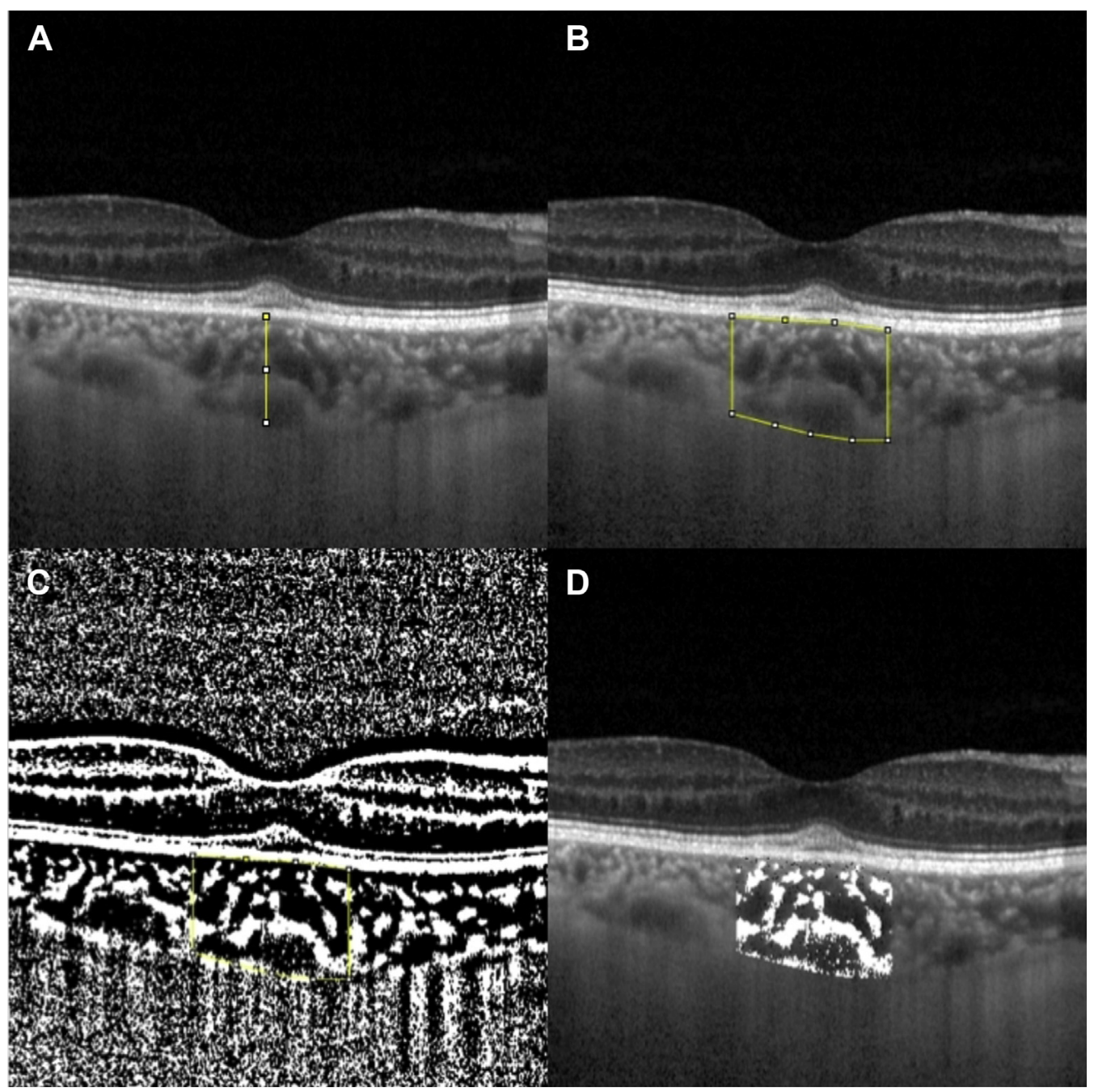

FIGURE 1. Subfoveal choroidal thickness (SFCT) and choroidal vascularity index (CVI) measurements. A. SFCT measurement on 8-bit spectral domain optical coherence tomography (SD-OCT) image. Vertical distance from the outer portion of the hyperreflective line corresponding to the retina pigment epithelium (RPE)-Bruch membrane complex to the hyperreflective line of the inner surface of the sclera was measured. B. 1500- $\mu \mathrm{m}$ subfoveal choroidal area centered at the fovea. C. Segmented OCT image using modified image binarization approach. D. Luminal (black) and stromal (white) parts are shown.

\section{MATERIALS AND METHODS}

THIS STUDY WAS APPROVED BY HACETTEPE UNIVERSITY Medical Faculty Ethical Committee and was conducted in accordance with the Declaration of Helsinki.

In this cross-sectional case-control study, SD-EDIOCT images of 19 eyes of 19 consecutive patients with angiographically proven CCF admitted to our Interventional Neuroradiology department during a 12-month period from March 2017 to March 2018 and 19 eyes of 19 age- and sex-matched healthy control subjects were included. SD-EDI-OCT images of subjects with concomitant macular or choroidal abnormalities, high myopia or hypermetropia ( $>6$ diopters), and images with poor quality were excluded from the analysis. Clinical data of the CCF patients were gathered from the hospital information system. Age, sex, angiographic findings including the venous drainage pattern, and treatment history were noted.

The patient group was divided according to CCF venous drainage pattern as anterior and posterior. Clinically affected eyes of the patient group with A-CCF and only one eye of P-CCF and the control subjects were included. All of the affected eyes in the A-CCF group had also ipsilateral ophthalmic venous drainage of the fistula.

SD-EDI-OCT (Heidelberg Spectralis; Heidelberg Engineering, Heidelberg, Germany) images of 38 eyes were analyzed for their central foveal thickness (CFT), subfoveal choroidal thickness (SFCT), CVI, and TI. The images were viewed and measured with the contained Heidelberg Eye Explorer software (Heidelberg Eye Explorer version 1.8.6.0; Heidelberg Engineering). The same 2 masked 


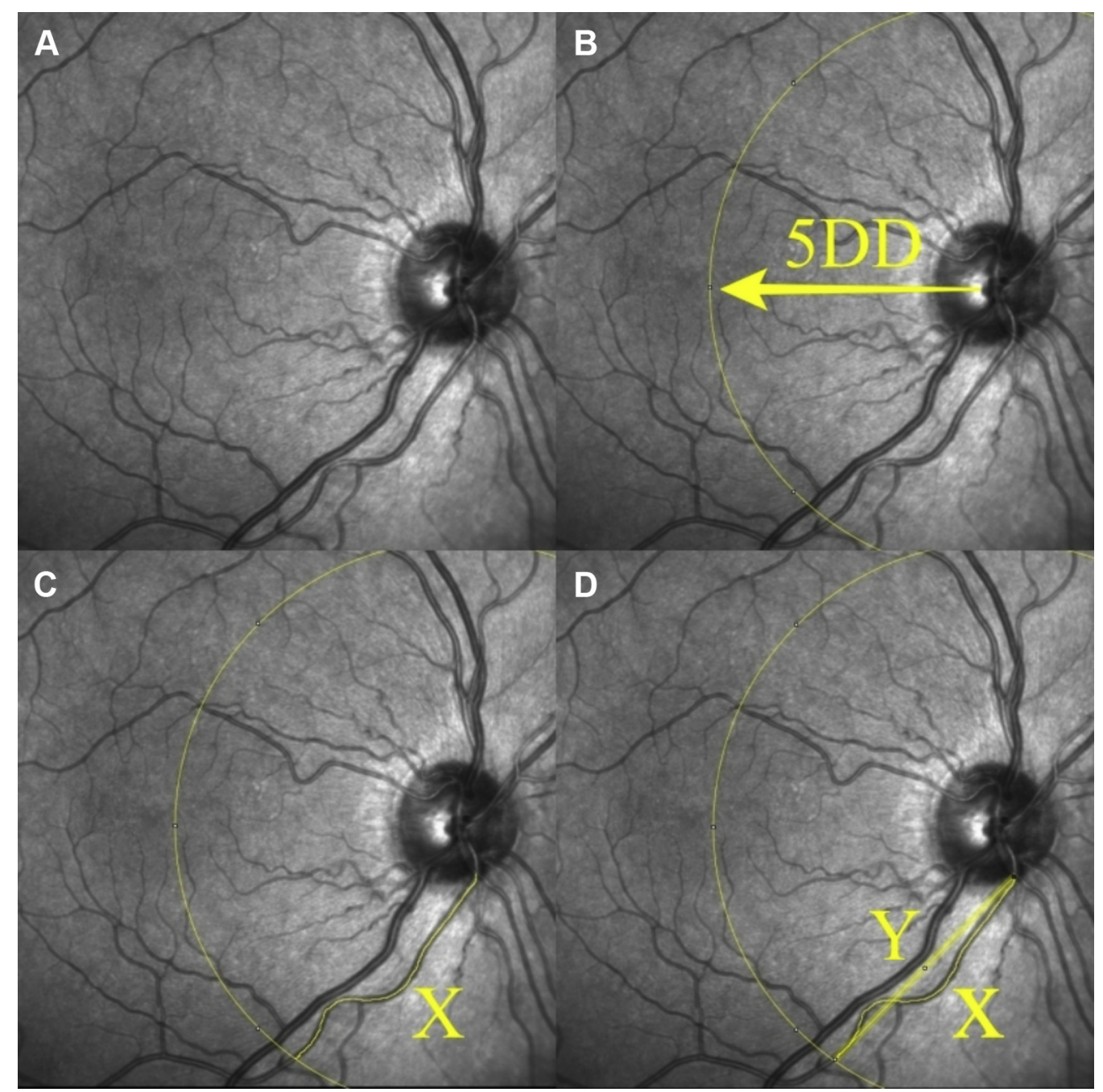

FIGURE 2. Tortuosity index (TI) measurements. A. Scale adjusted infrared fundus image. B. A circle with a diameter of 5-disc diameters (DDs) was drawn centered on the optic disc. C. X shows vessel tracing between disc margin and circle margin in the temporal area. D. Y shows the shortest length between disc margin and circle margin in the temporal area.

independent graders, who were both ophthalmologists, performed all of the measurements.

CFT was measured from the vitreomacular interference to the hyperreflective margin of the retina pigment epithelium (RPE)-Bruch membrane complex. Choroidal thickness was measured subfoveally on the transfoveolar scan, using the manual caliper function of the software. The SFCT was defined as the vertical distance from the outer portion of the hyperreflective line corresponding to the RPE-Bruch membrane complex to the hyperreflective line of the inner surface of the sclera (Figure 1, A).

CVI was measured as described in detail by Agrawal and associates $^{13}$ - using $750 \mu \mathrm{m}$ of each side of the central point of the fovea, a $1500-\mu \mathrm{m}$ area was used to measure CVI (Figure 1, B). Later on, the images were changed to 8-bit grayscale in order to perform the autothreshold function (Figure 1, C). After using the Niblack method for autothreshold, images were binarized to luminal and stromal areas (Figure 1, D). CVI, which is also equal to the luminal area percentage, was calculated as the proportion of luminal choroidal area to total choroidal area. Stromal area percentage (SAP), which is the remaining area, is also calculated as the proportion of stromal choroidal area to total choroidal area. Luminal to stromal ratio (LSR) is another parameter that could be calculated by dividing CVI by SAP.

TI measurements were performed as described by Mohsenin and associates. ${ }^{14}$ IR images taken by SD-OCT were used for TI measurements. IR images were opened in Fiji software and scale was adjusted for image scale. Optic disc center was marked and a 5-disc diameter circle was drawn by software automatically. After that, interested vessel was traced from its start point at disc margin till the end point at circle margin in temporal area and measured which is called as X. Shortest length measured between interested vessel's start point at disc margin and 
TABLE 1. Central Foveal Thickness (CFT) and Subfoveal Choroidal Thickness (SFCT) Values of the Anterior Carotid Cavernous Fistula (A-CCF) and Posterior Carotid Cavernous Fistula (P-CCF) Groups and the Control Group (CG)

\begin{tabular}{|c|c|c|c|c|c|c|}
\hline Region & A-CCF, $\bar{X} \pm \sigma(\mu \mathrm{m})$ & P-CCF, $\bar{X} \pm \sigma(\mu \mathrm{m})$ & $\mathrm{CG}, \overline{\mathrm{X}} \pm \sigma(\mu \mathrm{m})$ & $P$ Value $^{a}$ & $P$ Value $^{b}$ & $P$ Value $^{C}$ \\
\hline CFT & $200.57 \pm 31.89$ & $206.99 \pm 19.29$ & $218.68 \pm 19.91$ & .626 & .067 & .329 \\
\hline SFCT & $395.21 \pm 111.69$ & $246.84 \pm 94.12$ & $280.79 \pm 111.36$ & .039 & .006 & .554 \\
\hline \multicolumn{7}{|c|}{$\begin{array}{l}\text { A-CCF }=\text { anterior carotid cavernous fistu } \\
\text { SFCT = subfoveal choroidal thickness. } \\
{ }^{a} P \text { value between A-CCF and P-CCF. } \\
{ }^{b} P \text { value between A-CCF and CG. } \\
{ }^{c} P \text { value between P-CCF and CG. }\end{array}$} \\
\hline
\end{tabular}

end point at circle margin in temporal area was measured which is called as Y (Figure 2). TI calculations were done by dividing $\mathrm{X}$ value by $\mathrm{Y}$ value. TI calculations were performed for inferior temporal vein (IVTI), superior temporal vein (SVTI), and total venular tortuosity (VTTI) was calculated.

All the measurements were compared between the patient and the control group (CG). Normality of the data was assessed by Shapiro-Wilk Normality Test. Data which is found to have normal distribution was analyzed with parametric tests and data which is found not to have normal distribution was analyzed with non-parametric tests. $P<.05$ was taken as significant at all analysis. Mean and standard deviation were used to define continuous variables and percentage was used to define categorical variables. Student's $t$ test was used for normally distributed data and Mann Whitney $U$ test was used for the data that is not distributed normally.

\section{RESULTS}

THERE WERE A TOTAL OF 19 PATIENTS WITH ANGIOGRAPHically proven CCF in the study group (9 Female, 10 male). 15 of $19 \mathrm{CCF}(78.9 \%)$ had anterior, $4(21.1 \%)$ had posterior drainage. There were 9 females and 6 males in the A-CCF group and 1 female and 3 males in the P-CCF group. Mean age of subjects was $54.27 \pm 17.87$ years (range: $20-79$ years) in the A-CCF and $55.0 \pm 18.0$ years (range: 30-71years) in the P-CCF group.

Mean CFT was $200.57 \pm 31.89 \mu \mathrm{m}$ for A-CCF group, $206.99 \pm 19.29 \mu \mathrm{m}$ for P-CCF and $218.68 \pm 19.91 \mu \mathrm{m}$ for the CG. The differences in mean CFT values were statistically insignificant (Table 1, Figure 3).

The mean SFCT of the A-CCF group (395.21 $\pm 111.69 \mu \mathrm{m})$ was found to be significantly higher than both the P-CCF $(246.84 \pm 94.12 \mu \mathrm{m})$ and the CG $(280.79 \pm 111.36 \mu \mathrm{m})(P=.039$ and $P=.006$ respectively) (Table 1, Figure 3 ). However, there was no significant difference between P-CCF and CG $(P=.554)$ (Table 1, Figure 3).
The CVI, SAP, and LSR parameters were significantly different in the A-CCF group compared to CG. SAP was found to be lower, and CVI and LSR were found to be higher in the A-CCF group compared to CG $(P=.033$, $P=.033$, and $P=.027$ ) (Table 2 , Figure 4 ). In contrast, there was no significant difference between the P-CCF group and the CG for these parameters (Table 2, Figure 4).

All of the TI values, including IVTI, SVTI, and VTTI, of the A-CCF were significantly higher compared to the CG (Table 3), whereas there was no difference between the P-CCF and the CG for any of these parameters (Table 3).

Sample SD-EDI-OCT images of the CG, preprocedure, and 6-month follow-up images of the A-CCF group demonstrating the SFCT, CVI, and TI measurements are given in Figures 5-7.

\section{DISCUSSION}

MISDIAGNOSIS OR DELAY IN DIAGNOSIS OF CCF IS COMMON because of its diverse clinical manifestations and the rarity of the disease. Ophthalmologists are frequently the first clinicians encountering these cases. CCFs are commonly misdiagnosed as conjunctivitis, thyroid eye disease, or idiopathic orbital inflammatory disease because clinical manifestations may not be so apparent especially in patients with a dural CCF. Timely intervention in CCF is very important to prevent morbidity, including vision loss. SD-EDI-OCT is a fast and noninvasive tool that may aid in the diagnosis and follow-up of these cases. Our study aimed to demonstrate the changes in the choroidal and retinal vessels in 3 ways: choroidal and retinal thickness measurements, CVI measurements, and TI measurements.

Since the invention of OCT by Huang and associates at the Massachusetts Institute of Technology, imaging of ophthalmologic conditions improved progressively. ${ }^{15}$ Owing to the limitations of this very first OCT, SD-OCT became available in the last decade, providing us with the advantage of higher resolution, faster image acquisition, and additional improvements such as eye tracking technologies. ${ }^{16,17}$ EDI-OCT has further improved the level 
A

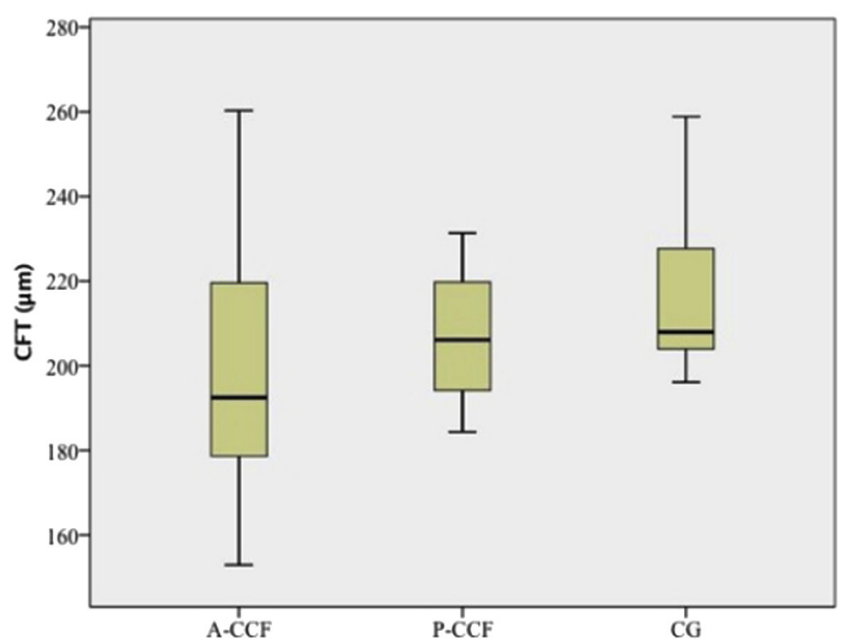

B

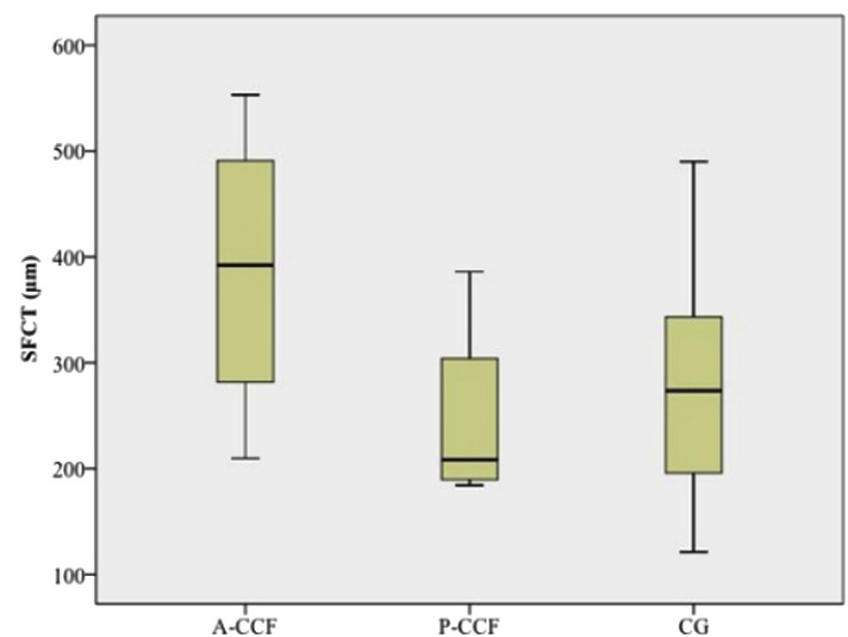

FIGURE 3. Central foveal thickness (CFT) and subfoveal choroidal thickness (SFCT) graphics of the anterior carotid cavernous fistula (A-CCF), posterior carotid cavernous fistula (P-CCF) groups, and the control group (CG). A. No difference was noted between groups for the CFT. B. Mean SFCT of A-CCF is significantly higher than both P-CCF and CG $(P=.039$ and $P=.006$ respectively).

TABLE 2. Choroidal Vascularity Index (CVI) Parameters of the Anterior Carotid Cavernous Fistula (A-CCF) and Posterior Carotid Cavernous Fistula (P-CCF) Groups and the Control Group (CG)

\begin{tabular}{|c|c|c|c|c|c|c|}
\hline & $\mathrm{A}-\mathrm{CCF}, \overline{\mathrm{X}} \pm \sigma$ & $\mathrm{P}-\mathrm{CCF}, \overline{\mathrm{X}} \pm \sigma$ & $\mathrm{CG}, \overline{\mathrm{X}} \pm \sigma$ & $P$ Value $^{a}$ & $P$ Value $^{b}$ & $P$ Value $^{C}$ \\
\hline CVI & $68.97 \pm 4.81$ & $70.96 \pm 4.81$ & $65.66 \pm 3.37$ & .496 & .033 & .111 \\
\hline SAP & $31.03 \pm 4.81$ & $29.04 \pm 4.81$ & $34.34 \pm 3.37$ & .496 & .033 & .111 \\
\hline TCA, $\mu \mathrm{m}^{2}$ & $423029.4 \pm 159546.7$ & $284877.45 \pm 113687.38$ & $437386.48 \pm 165612.22$ & .092 & .800 & .067 \\
\hline $\mathrm{LCA}, \mu \mathrm{m}^{2}$ & $286400.1 \pm 91758.63$ & $198754.29 \pm 67325.11$ & $286216.59 \pm 105110.56$ & .075 & .996 & .075 \\
\hline $\mathrm{SCA}, \mu \mathrm{m}^{2}$ & $136629.3 \pm 69122.3$ & $86123.16 \pm 46736.26$ & $151169.89 \pm 63254.32$ & .129 & .532 & .059 \\
\hline
\end{tabular}

$\mathrm{A}-\mathrm{CCF}=$ anterior carotid cavernous fistula; $\mathrm{CG}=$ control group; $\mathrm{CVI}=$ choroidal vascularity index; LCA = luminal choroidal area; LSR = luminal to stromal ratio; P-CCF = posterior carotid cavernous fistula; SAP = stromal area percentage; SCA = stromal choroidal area; $\mathrm{TCA}=$ total choroidal area.

${ }^{a} P$ value between A-CCF and P-CCF.

${ }^{b} P$ value between $A-C C F$ and $C G$.

${ }^{c} P$ value between $P-C C F$ and $C G$.

of imaging so that choroidal measurements can be made from an OCT image. ${ }^{18}$ The ability of quantifying choroidal parameters was utilized in 3 cases of CCF in the literature. $^{10-12} \mathrm{CCF}$ triggers some basic hemodynamic changes in the ocular structures such as an increase in episcleral venous pressure and decrease in arterial perfusion-hence nourishment. ${ }^{19}$ These findings can cause central venous thrombosis in return. ${ }^{19}$ The increased venous pressure can be said to cause some choroidal changes that were not well demonstrated prior to the EDI-OCT technology. Recently, Shinohara and associates were the first to describe the relationship between SFCT and CCF in their case report. ${ }^{11}$ The 3 case reports in the literature reported increased choroidal thickness in patients with CCF that eventually decreased after the closure of the fistula. They all suggested that SFCT could be a useful index for measuring the venous congestion effects of $\mathrm{CCF}^{10-12}$ The preliminary findings in these case reports were suggestive for a choroidal congestion and CCF relationship. Yet the available data is at the level of case reports only, and solely the choroidal thickness was used as an indicator of congestion. In our study, we aimed to further scrutinize the pathophysiology underlying these findings and examine the clinical use of these findings in a cohort of patients.

Mean CFT difference was not previously investigated in patients with CCF and we found that there was no significant difference between any group despite previous 
A

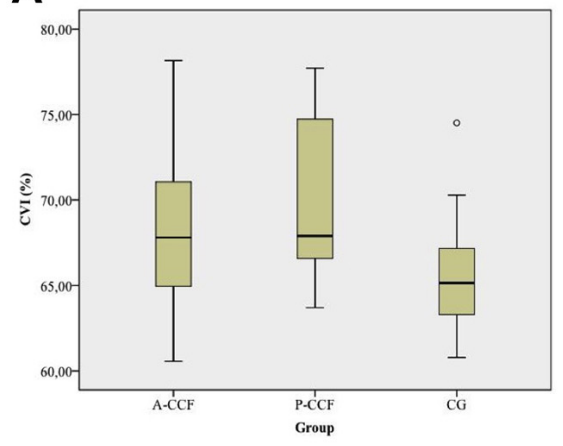

D

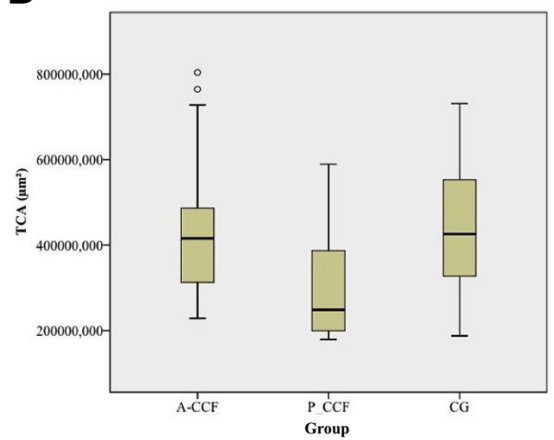

B

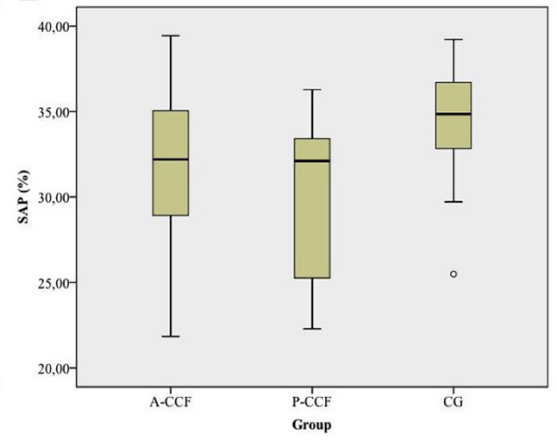

E

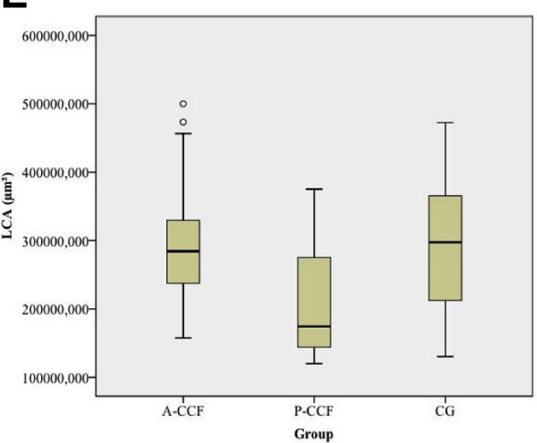

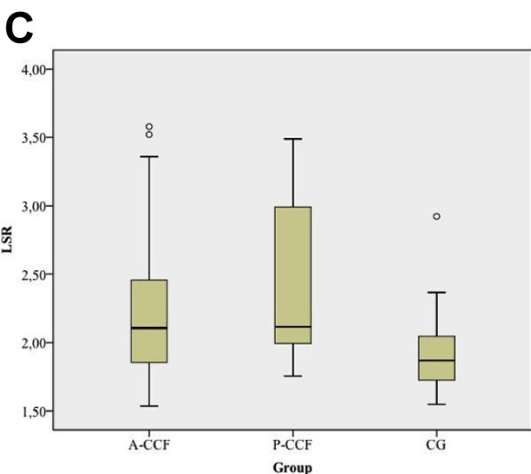

$\mathbf{F}$

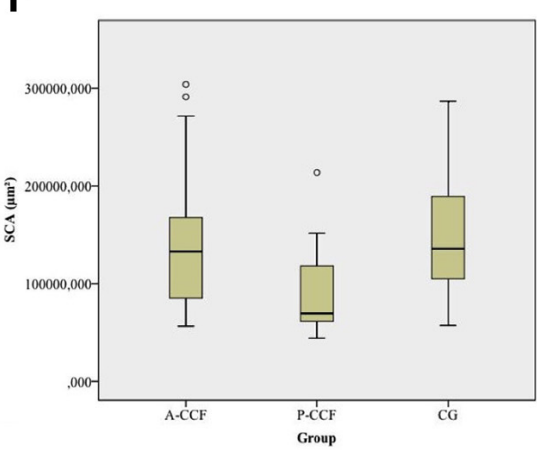

FIGURE 4. Choroidal vascularity index (CVI) graphics of the anterior carotid cavernous fistula (A-CCF), posterior carotid cavernous fistula (P-CCF) groups, and the control group (CG). A. CVI of the A-CCF is noted to be significantly higher than the CG $(P=.033)$. B. Stromal area percentage (SAP) value of the A-CCF is shown to be significantly lower than the CG $(P=.033)$. C. Luminal to stromal ratio (LSR) of the A-CCF is significantly higher than the CG $(P=.027)$. D-F. None of the total choroidal area (TCA), luminal choroidal area (LCA), and stromal choroidal area (SCA) parameters are noted to be different in between groups.

TABLE 3. Tortuosity Index (TI) Parameters of the Anterior Carotid Cavernous Fistula (A-CCF) and Posterior Carotid Cavernous Fistula (P-CCF) Groups and the Control Group (CG)

\begin{tabular}{lcccccc}
\hline TI & $\mathrm{A}-\mathrm{CCF}, \overline{\mathrm{X}} \pm \sigma$ & $\mathrm{P}-\mathrm{CCF}, \overline{\mathrm{X}} \pm \sigma$ & $\mathrm{CG}, \overline{\mathrm{X}} \pm \sigma$ & $P$ Value $^{a}$ & $P$ Value $^{b}$ & $P$ Value \\
\hline IVTI & $1.1302 \pm 0.0802$ & $1.1005 \pm 0.0684$ & $1.0469 \pm 0.0466$ & .488 & .001 & .216 \\
SVTI & $1.1133 \pm 0.0843$ & $1.0418 \pm 0.0172$ & $1.0327 \pm 0.0336$ & .117 & .001 & .455 \\
VTTI & $1.1217 \pm 0.0742$ & $1.0711 \pm 0.0416$ & $1.0398 \pm 0.0266$ & .108 & $<.001$ & .231 \\
\hline
\end{tabular}

A-CCF = anterior carotid cavernous fistula; $\mathrm{CG}=$ control group; IVTI = inferior temporal vein tortuosity index; P-CCF = posterior carotid cavernous fistula; SVTI = superior temporal vein tortuosity index; VTTI = total venular tortuosity index.

${ }^{a} P$ value between $A-C C F$ and $P-C C F$.

${ }^{b} P$ value between $A-C C F$ and $C G$.

${ }^{c} P$ value between $P-C C F$ and $C G$.

studies that mentioned atrophic effects of long-standing CCF. ${ }^{11}$ The mean SFCT of the A-CCF was found to be significantly higher than both the CG and P-CCF, which is most likely due to choroidal congestion. There was no significant difference between P-CCF and CG. This finding is to be expected because the drainage of $\mathrm{P}-\mathrm{CCF}$ is mainly to the inferior petrosal sinus and not to the ophthalmic vein. Thus, these fistulas do not cause choroidal congestion.
The choroidal thickness measurements can give an inspiring opinion about the congestive effects of CCF on choroid, but they are not able to give sufficient information about the underlying mechanism, including the changes in the luminal or stromal portion of the choroid. Also, choroidal thickness may not be a reliable parameter in clinical research or patient care because there are several physiologic factors influencing its measurement, such as diurnal variation, gender, and age. ${ }^{20}$ Recently Agrawal and 


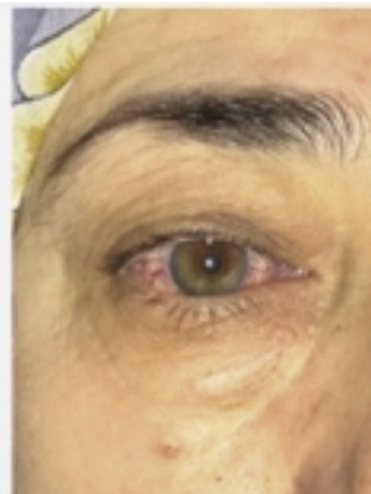

A

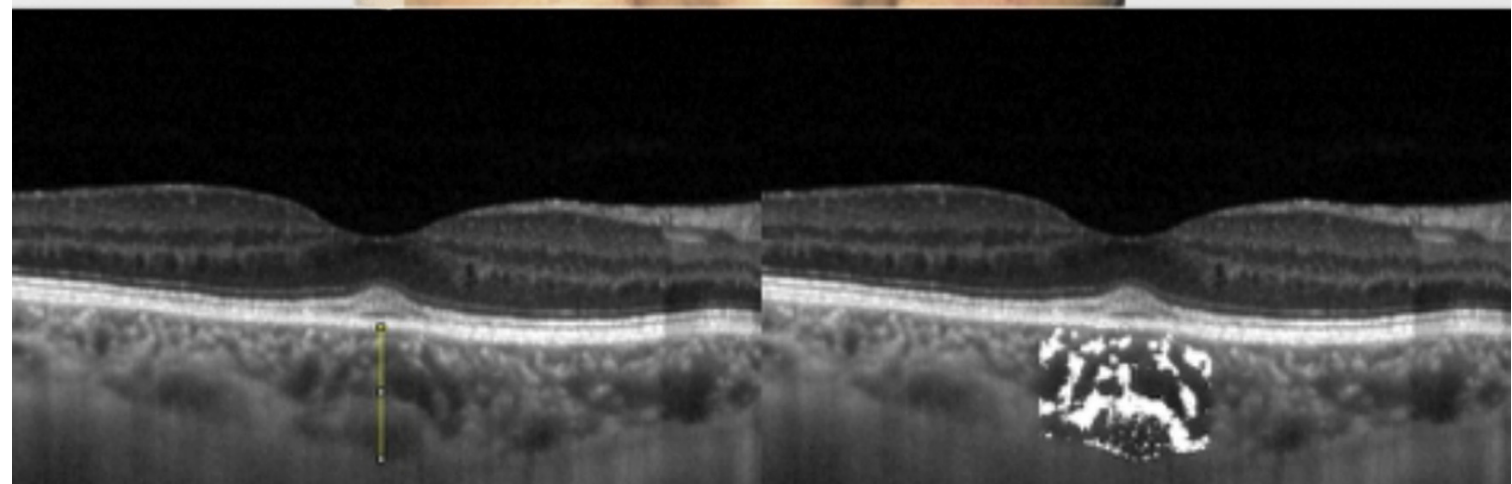

B : C

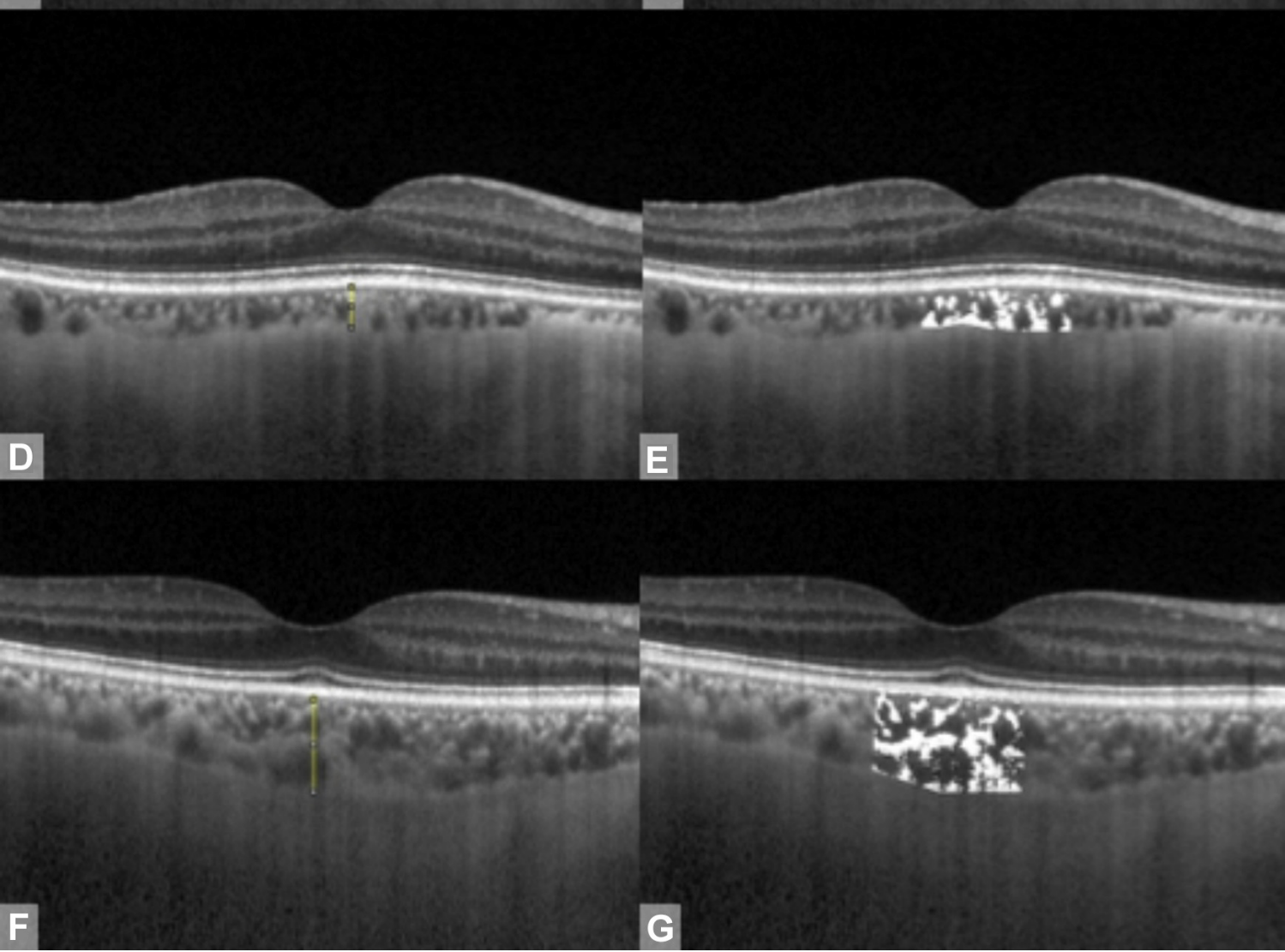

FIGURE 5. Clinical and spectral domain enhanced-depth imaging optical coherence tomography (SD-EDI-OCT) images of a patient with an anterior carotid cavernous fistula (A-CCF) before and after treatment (A-C, F, G) and a subject from the control group (CG) $(D, E)$. A. Clinical photo of the patient with A-CCF affecting the right eye. B. SD-EDI-OCT image of the right eye of the same patient with a subfoveal choroidal thickness (SFCT) measurement of $349 \mu \mathrm{m}$. C. Binarized image of the right eye of the same patient with a 


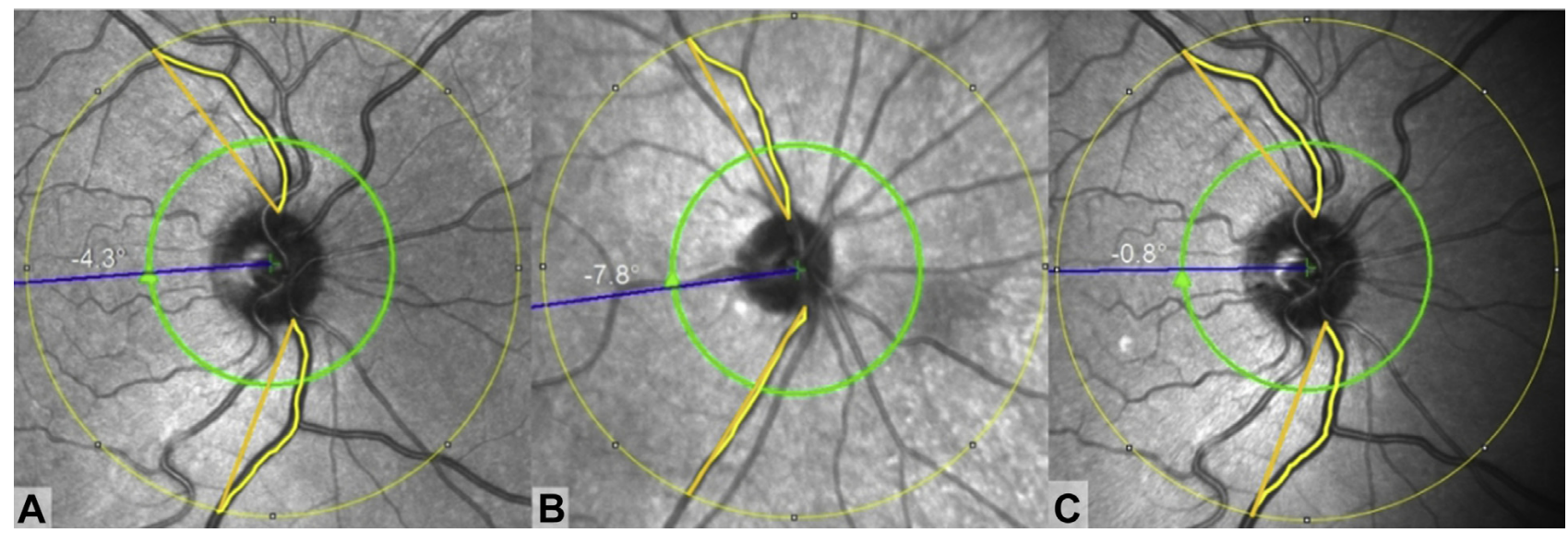

FIGURE 6. Tortuosity index (TI) measurements of a patient with an anterior carotid-cavernous fistula (A-CCF) before and after treatment. A. Scale-adjusted infrared (IR) fundus image of the right eye of a patient from the A-CCF group with a total venular tortuosity (VTTI) measurement of 1.1364. B. Scale-adjusted IR fundus image of the right eye of a subject from the control group (CG) with a VTTI measurement of 1.0381. C. Scale-adjusted IR fundus image of the right eye of the same subject 6 months after treatment with a VTTI measurement of 1.1190 .

associates proposed a new quantitative parameter, namely, CVI, to assess the vascular status of the choroid through image binarization of SD-EDI-OCT images in healthy eyes. ${ }^{13}$ In this study, CVI showed lesser variability and was influenced by fewer physiologic factors compared to the choroidal thickness measurements. As the choroid is primarily a vascular structure, authors stated that this new vascular index might elucidate the role of vascular processes within the choroid in disease development and progression. CVI gave sufficient causative information for choroidal changes in diabetes mellitus, central serous chorioretinopathy, adult macular degeneration, panuveitis and Vogt-Koyanagi-Harada syndrome. ${ }^{13,21-27}$ Thus, we measured the CVI parameters for CCF subjects in order to better illuminate the effects of the fistula on the choroid. To our knowledge, there is no study in the literature investigating CVI parameters in patients with CCF. Patients with A-CCF were found to have significantly higher CVI, and thus higher luminal and LSR values, compared to the CG. Corresponding to this finding, SAP values were lower in the A-CCF group. In contrast, these parameters were similar in P-CCF compared to the control group. These findings suggest that increased venous pressure due to CCF increases the percentage of the luminal component within the choroid, causing increases in choroidal thickness.

Retinal venular dilatation and tortuosity are commonly observed findings in patients with CCF. Over the years, tortuosity has been evaluated subjectively by clinicians, but because of high interobserver variability, the sensitivity and repeatability of this evaluation is limited. ${ }^{28}$ Quantitative assessment of tortuosity has been reported in different retinal pathologies, ${ }^{29-32}$ using a single distance measurement or a combination of measurements. ${ }^{29,30,32,33}$ To our knowledge, this is also the first study investigating retinal venular tortuosity in patients with CCF by tortuosity index analysis. We found that venous tortuosity in all regions were higher in A-CCF compared to the CG. This finding was not observed in the P-CCF group.

$\mathrm{CCF}$, an uncommon, potentially vision-threatening disease, can masquerade as conjunctivitis or other common ocular conditions, precluding a chance for an early diagnosis. A high index of clinical suspicion is required in patients presenting with a red eye, especially in cases of dural fistulas where the clinical features are often not diagnostic. Although carotid angiogram is the criterion standard in the diagnosis of CCF, a noninvasive imaging method supporting the first suspicion of the clinician would be valuable before ordering more costly and invasive imaging studies. We believe CVI and TI evaluation in addition to choroidal thickness measurements may meet this need.

One limitation of our study is the small sample size, despite the fact that all consecutive patients were enrolled in a tertiary referral center. This is due to low incidence of carotid-cavernous fistula. Additional classification of fistulae based on drainage pattern further diminishes the group sizes. Thus, future investigations with a larger

choroidal vascularity index (CVI) measurement of 68.01. D. SD-EDI-OCT image of a subject from the CG with an SFCT measurement of $125 \mu \mathrm{m}$. E. Binarized image of the same subject from CG with a CVI measurement of 64.03. F. SD-EDI-OCT image of the same patient with A-CCF 6 months after the closure of the fistula with an SFCT measurement of $290 \mu \mathrm{m}$. G. Binarized image of the same patient with A-CCF 6 months after treatment with a CVI measurement of 62.60 . 


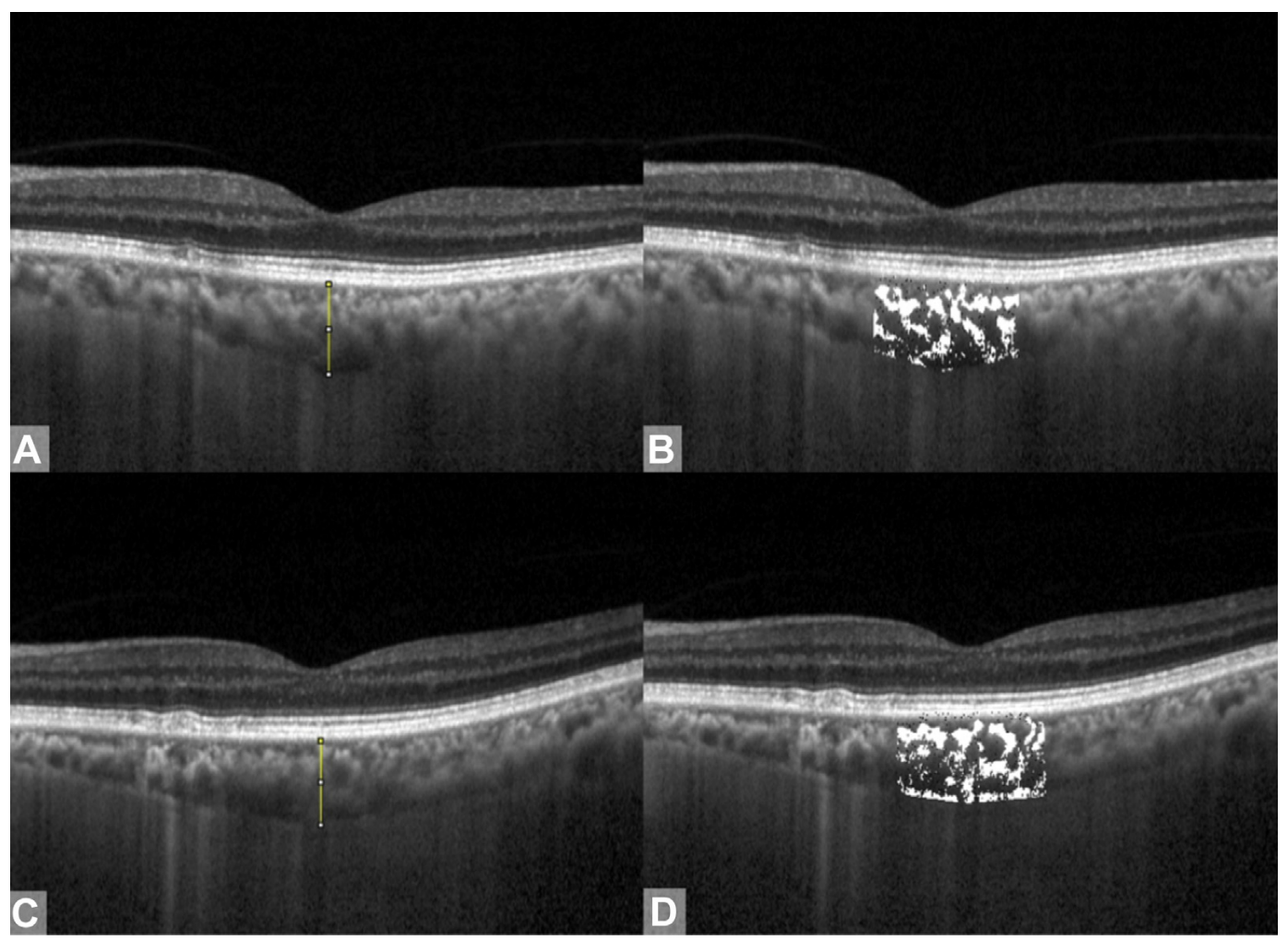

FIGURE 7. Subfoveal choroidal thickness (SFCT) and choroidal vascularity index (CVI) measurements of a patient with an anterior carotid cavernous fistula (A-CCF) before and after treatment. A. Spectral domain enhanced-depth imaging optical coherence tomography (SD-EDI-OCT) image of the left eye of the patient from the A-CCF group with an SFCT measurement of $290 \mu \mathrm{m}$. B. Binarized image of the left eye of the same patient with a CVI measurement of 67.86. C. SD-EDI-OCT image of the same subject 6 months after closure of the fistula with an SFCT measurement of $278 \mu \mathrm{m}$. D. Binarized image of the same subject 6 months after the treatment with a CVI measurement of 64.54 .

sample size are needed. Another limitation is the absence of a postprocedure data set pertaining to the temporal evolution of our findings. As we were initially planning our study, we were quite uncertain about the clinical utility of these EDI-OCT indices. So we did not structure the study to investigate the changes of these parameters after treatment. However, postprocedure findings available in 3 of our patients (Figures 5-7) suggest that the aforementioned SD-EDI-OCT indices are promising for clinical follow-up. Further studies are needed to determine the utility of EDI-OCT during the clinical follow-up of patients with CCF, especially in those cases with a possible residual or recurrent fistula, the diagnosis of which frequently entails an invasive cerebral angiogram.

In conclusion, we demonstrated increased choroidal thickness, choroidal vascularity and tortuosity indices in CCFs with anterior drainage by SD-EDI-OCT, which is a quick, noninvasive, readily available tool in many clinics. Although the number of cases with posterior drainage is low in our study, these indices seem to correlate well with the angiographic drainage pattern of the CCFs. The aforementioned parameters are candidates to be both evaluative and diagnostic tools for the CCFs and may also provide guidance during the follow-up.

\section{REFERENCES}

1. Tan AC, Farooqui S, Li X, et al. Ocular manifestations and the clinical course of carotid cavernous sinus fistulas in Asian patients. Orbit 2014;33(1):45-51.
2. Kiyosue H, Hori Y, Okahara M, et al. Treatment of intracranial dural arteriovenous fistulas: current strategies based on location and hemodynamics, and alternative techniques of transcatheter embolization. Radiographics 2004;24(6): 1637-1653. 
3. Kim MS, Han DH, Kwon OK, Oh CW, Han MH. Clinical characteristics of dural arteriovenous fistula. J Clinical Neurosci 2002;9(2):147-155.

4. Debrun GM. Treatment of traumatic carotid-cavernous fistula using detachable balloon catheters. AJNR Am J Neuroradiol 1983;4(3):355-356.

5. Miller NR. Dural carotid-cavernous fistulas: epidemiology, clinical presentation, and management. Neurosurg Clin N Am 2012;23(1):179-192.

6. Talks SJ, Salmon JF, Elston JS, Bron AJ. Cavernous-dural fistula with secondary angle-closure glaucoma. Am J Ophthalmol 1997;124(6):851-853.

7. Fiore PM, Latina MA, Shingleton BJ, Rizzo JF, Ebert E, Bellows AR. The dural shunt syndrome. I. Management of glaucoma. Ophthalmology 1990;97(1):56-62.

8. Alm A, Bill A. Ocular and optic nerve blood flow at normal and increased intraocular pressures in monkeys (Macaca irus): a study with radioactively labelled microspheres including flow determinations in brain and some other tissues. Exp Eye Res 1973;15(1):15-29.

9. Nickla DL, Wallman J. The multifunctional choroid. Prog Retin Eye Res 2010;29(2):144-168.

10. Gonzalez Martin-Moro J, Sales-Sanz M, OblancaLlamazares N, Bustos-Garcia A, Mendez-Cendon JC, Fandino E. Choroidal thickening in a case of carotid cavernous fistula. Orbit 2018;37(4):306-308.

11. Shinohara Y, Kashima T, Akiyama H, Kishi S. Alteration of choroidal thickness in a case of carotid cavernous fistula: a case report and a review of the literature. BMC Ophthalmol 2013;13:75

12. Rey A, Castillo L, Dyrda A, Maseras X, Jurgens I. Subfoveal choroidal thickness changes in carotid cavernous fistula following spontaneous resolution. BMC Ophthalmol 2016; $16: 63$.

13. Agrawal R, Gupta P, Tan KA, Cheung CM, Wong TY, Cheng CY. Choroidal vascularity index as a measure of vascular status of the choroid: measurements in healthy eyes from a population-based study. Sci Rep 2016;6:21090.

14. Mohsenin A, Mohsenin V, Adelman RA. Retinal vascular tortuosity in obstructive sleep apnea. Clin Ophthalmol 2013; 7:787-792.

15. Huang D, Swanson EA, Lin CP, et al. Optical coherence tomography. Science 1991;254(5035):1178-1181.

16. Nassif NA, Cense B, Park BH, et al. In vivo high-resolution video-rate spectral-domain optical coherence tomography of the human retina and optic nerve. Opt Express 2004;12(3); $367-376$.

17. Murthy RK, Haji S, Sambhav K, Grover S, Chalam KV. Clinical applications of spectral domain optical coherence tomography in retinal diseases. Biomed J 2016;39(2):107-120.

18. Margolis R, Spaide RF. A pilot study of enhanced depth imaging optical coherence tomography of the choroid in normal eyes. Am J Ophthalmol 2009;147(5):811-815.
19. Jørgensen JS, Guthoff R. Ophthalmoscopic findings in spontaneous carotid cavernous fistula: an analysis of 20 patients. Graefes Arch Clin Exp Ophthalmol 1988;226(1):34-36.

20. Gupta P, Jing T, Marziliano P, et al. Distribution and determinants of choroidal thickness and volume using automated segmentation software in a population-based study. Am J Ophthalmol 2015;159(2):293-301.e3.

21. Sonoda S, Sakamoto T, Yamashita T, et al. Luminal and stromal areas of choroid determined by binarization method of optical coherence tomographic images. Am J Ophthalmol 2015;159(6):1123-1131.e1.

22. Tan KA, Agrawal R. Luminal and stromal areas of choroid determined by binarization method of optical coherence tomographic images. Am J Ophthalmol 2015;160(2):394.

23. Tan KA, Laude A, Yip V, Loo E, Wong EP, Agrawal R. Choroidal vascularity index-a novel optical coherence tomography parameter for disease monitoring in diabetes mellitus? Acta Ophthalmol 2016;94:e612-e616.

24. Agrawal R, Chhablani J, Tan KA, Shah S, Sarvaiya C, Banker A. Choroidal vascularity index in central serous chorioretinopathy. Retina 2016;36(9):1646-1651.

25. Koh LHL, Agrawal R, Khandelwal N, Sai Charan L, Chhablani J. Choroidal vascular changes in age-related macular degeneration. Acta Ophthalmol 2017;95(7):e597-e601.

26. Agrawal R, Salman M, Tan KA, et al. Choroidal vascularity index (CVI) — a novel optical coherence tomography parameter for monitoring patients with panuveitis? PLoS One 2016; 11(1):e0146344.

27. Agrawal R, Li LK, Nakhate V, Khandelwal N, Mahendradas P. Choroidal vascularity index in Vogt-Koyanagi-Harada disease: an EDI-OCT derived tool for monitoring disease progression. Transl Vis Sci Technol 2016;5(4):7.

28. Kagan A, Aureli E, Dobree J. A note on signs in the fundus oculi and arterial hypertension: conventional assessment and significance. Bull World Health Organ 1966;34(6):955-960.

29. Sasongko MB, Wong TY, Nguyen TT, Cheung CY, Shaw JE, Wang JJ. Retinal vascular tortuosity in persons with diabetes and diabetic retinopathy. Diabetologia 2011;54(9):2409-2416.

30. Koreen S, Gelman R, Martinez-Perez ME, et al. Evaluation of a computer-based system for plus disease diagnosis in retinopathy of prematurity. Ophthalmology 2007;114(12):e59-e67.

31. Hughes AD, Martinez-Perez E, Jabbar AS, et al. Quantification of topological changes in retinal vascular architecture in essential and malignant hypertension. J Hypertens 2006; 24(5):889-894.

32. Gelman R, Martinez-Perez ME, Vanderveen DK, Moskowitz A, Fulton AB. Diagnosis of plus disease in retinopathy of prematurity using Retinal Image multiScale Analysis. Invest Ophthalmol Vis Sci 2005;46(12):4734-4738.

33. Alam M, Thapa D, Lim JI, Cao D, Yao X. Quantitative characteristics of sickle cell retinopathy in optical coherence tomography angiography. Biomed Opt Express 2017;8(3): $1741-1753$. 